\title{
Development of an app as a tool to support research and the \\ prevention of osteoporosis
}

Hélio Luiz Fernando Bernardi' Luciana Branco da Motta ${ }^{2}$

\section{Abstract}

Objective: to develop an app for the investigation and prevention of osteoporosis for use by health professionals. Method: the development of the app was performed in six steps: definition of the target audience; survey of validated osteoporosis risk assessment tools in Brazil; programming of the app through the progressive web; selection of data for the preparation of sections of the evaluation form and clinical recommendations; automated auditing and evaluation of the app by health professionals. Results: three screens were prepared for the app. These were based on the data extracted using the Osteorisk, Sapori and Frax tools for sociodemographic data (age, gender, weight, height and ethnicity), health (use of glucocorticoids, hormone replacement therapy, arthritis rheumatoid arthritis, secondary osteoporosis, previous low impact fractures, parents with a history of hip fractures) and health related behaviors (physical activity, alcohol intake and smoking). The appl followed the design pattern and functionalities of the osteoporosis adviser tool (OPAD). Regarding guidelines relating to clinical recommendations, the guidelines on osteoporosis and fall prevention in the elderly of the Ministry of Health and the Brazilian Society of Geriatrics and Gerontology, respectively, were taken as a basis. Conclusion: the app allows the early identification of patients presenting risk factors for osteoporosis and, based on these results, provides guidance on the preventive measures to be adopted, aiming at reducing complications resulting from fractures, hospitalizations, disabilities and deaths.

\footnotetext{
Universidade do Estado do Rio de Janeiro, Programa de pós-graduação em Telemedicina e Telessaúde. Rio de Janeiro, Rio de Janeiro, Brasil.

2 Universidade do Estado do Rio de Janeiro, Universidade Aberta da Terceira Idade, Núcleo de Atenção ao Idoso. Rio de Janeiro, Rio de Janeiro, Brasil.
}

Keywords: Aging. Osteoporosis. Decision Making, Computer Assisted. 


\section{INTRODUCTION}

Brazil is a large country that includes states with isolated municipal regions, a multiethnic population and an unequal supply of specialized medical services that hamper epidemiological studies into the accurate prevalence of osteoporosis and its impact on public health costs, limiting the management of the disease ${ }^{1}$. This situation encourages greater use of telecare and apps and decision support tools in this area, and in turn to more publications in the application of mobile technology in health line of research ${ }^{2}$.

The spread of information and communication technologies, with the global use of mobile devices and broadband expansion, has had major repercussions in the area of medicine. This technological configuration has enabled the development of web systems and specific applications for health professionals and patients. Examples include the Alzhe alert, which estimates the risk of a person developing Alzheimer's disease over the years, depending on their daily habits and activities; ${ }^{3}$; and ADep (Ayuda para depression in Spanish), a free access program on psychoeducation and cognitive-behavioral intervention for depression, produced in Mexico ${ }^{4}$.

In Brazil, other examples of these types of applications are Mobi-Frail (Frágil Mobi), which seeks to identify characteristics of frailty in the elderly ${ }^{5}$, and the Tech-Care (CuidarTech) "Foot Examination" app, a tool to assist in the assessment and classification of risk of developing diabetic foot for people with diabetes mellitus ${ }^{6}$.

One of the great challenges facing Brazil is the aging of its population. Osteoporosis and fractures due to frailty are highlighted by high costs to public health, negatively impacting the quality of life of the elderly. In Brazilian studies, the reported prevalence of osteoporosis among postmenopausal women ranges from $15.0 \%$ to $33.0 \%$. In general, these studies also show a high prevalence of all types of fractures due to bone fragility, varying from 11.0 to $23.8 \%$; and a moderate incidence of hip fractures in individuals over 50 years of age ${ }^{8}$.

An epidemiological study of fractures of the proximal third of the femur in elderly patients treated at a teaching hospital in the central region of São
Paulo showed that low-energy trauma was the cause of $92.9 \%$ of fractures, with an intra-hospital mortality rate of $7.1 \%{ }^{9}$. It is estimated that $15.0 \%$ to $30.0 \%$ of patients with hip fractures die in the first year, often as a result of fracture complications, such as infections, venous thrombosis and pressure ulcers; or associated conditions, especially cardiovascular in nature ${ }^{1}$

Aiming to avoid these complications, several European, North American and Asian studies have demonstrated the clinical relevance of assessment tools that can be used to identify individuals at a higher risk of developing osteoporosis. Some of these tools, such as the simple calculated osteoporosis estimate (Score) and the Belgian risk ratio for osteoporosis $(\mathrm{Osiris})^{10}$, indicate that the early identification of risk factors is the main goal in the introduction of effective osteoporosis prevention strategies $^{11}$, which were considered in the elaboration of the application in the present study, known as OSTEOGUIA (OSTEOGUIDE).

The objective of this study was to develop a progressive web app to assist health professionals in the assessment and prevention of osteoporosis with content based on risk assessment tools available in literature.

\section{METHOD}

A study was performed to develop a low-cost app that can be used by any health service and is easy to install on mobile devices.

The steps for building the app involved the definition of the target audience; a survey of validated osteoporosis risk assessment tools in Brazil; programming of the app through the progressive web; selection of data for the sections of the assessment form and clinical recommendations; automated auditing and evaluation of the app by health professionals.

\section{Target audience}

As osteoporosis is a disease associated with aging, and that approximately $75 \%$ of Brazilians do not 
have a private health plan $^{1}$, the target audience of the app was defined as health professionals working in the basic health, women's and geriatric areas of the Unified Health System (SUS).

\section{Survey of validated osteoporosis risk assessment} tools in Brazil

A survey of scientific publications was carried out in the Medline and Lilacs databases of the Virtual Health Library (VHL) and in the Capes journal portal, aiming to identify the validated risk assessment tools for osteoporosis in Brazil. The criteria for inclusion of articles were: publications available online, in Portuguese or English and published between 2005 and 2016. We excluded studies describing tools not evaluated for the Brazilian population. The descriptors used were: osteoporosis, risk factors, risk assessment and hip fractures. After the evaluation of the articles, three tools (Osteorisk, Sapori and Frax) were identified that served as basis for the creation of the app.

The Osteorisk tool is based on age and weight, and the evaluation of the Brazilian population sample showed an overall sensitivity to identify women with a high risk of osteoporosis of $86.5 \%$. The simplicity of the app, its low cost and the savings generated make this tool an excellent screening method to identify women who are at higher risk of osteoporosis ${ }^{12}$.

The Sapori tool identified that the main risk factors associated with low bone density and low impact fractures were: underweight; advanced age; white/Caucasian race/ethnicity; a family history of femoral fracture; current smoker and chronic use of glucocorticosteroids. Postmenopausal hormone therapy and regular physical activity played a protective role ${ }^{13}$.

The Frax tool has an algorithm that calculates the risk of the patient suffering a fracture due to bone fragility in the next ten years, correlating the risk factors with the result of the bone mineral density of the femoral neck, measured by bone densitometry. The clinical risk factors assessed are: age; gender; body mass index (BMI); previous fractures; family history of femoral fracture; prolonged corticotherapy; current smoker; ingestion of more than three units of alcoholic beverage per day; secondary osteoporosis and rheumatoid arthritis ${ }^{14}$.

\section{Progressive web app programming}

For programming the app open source technologies such as HTML, CCS, Java script and PHP were used. We chose to develop a progressive web app (PWA), a term used to denote a new software development methodology. Unlike traditional apps, a PWA can be seen as a hybrid evolution between regular web pages (or websites) and a mobile app. This new app model combines features offered by the most modern browsers with the advantages of using a mobile phone $\mathrm{e}^{15}$. Throughout the development of this application we tried to meet the precepts established in software engineering.

\section{Selection of data for preparation of the Assessment Form section and for clinical recommendations}

The use of risk assessment tools should consider the profile of their population, as genetic, racial and anthropometric differences contribute to explain divergences in the incidence and prevalence of low bone density and fractures in several countries around the world ${ }^{13}$. The selection of data for the preparation of the assessment form was based on the three validated osteoporosis risk assessment tools in Brazil (Osteorisk, Sapori and Frax).

The weight and age data common to the three tools were used in the patient risk classification for low bone density which was based on the algorithm calculation of the Osteorisk tool: $0.2 *$ [(Weight in $\mathrm{Kg}$ ) - (Age in years)]. Individuals are considered low risk when the result of the calculation is greater than 1 , medium risk when the value is between -2 and 1 and high risk when the value is less than -2 . This tool performs well, as shown in the work of Sen et al. ${ }^{11}$ and Steiner et al. ${ }^{12}$, with sensitivity of $86.5 \%$ in the identification of women at high risk for osteoporosis.

Family history, specifically parents with a history of femoral fracture, current smoking, chronic use of glucocorticoids and previous low impact fractures 
were identified as risk factors associated with low bone density by the Sapori and Frax tools and were incorporated into the app.

The use of glucocorticoids followed the Frax recommendation to indicate a positive result if current oral glucocorticoid use was identified for more than 3 months with a prednisolone dose of $5 \mathrm{mg}$ or more (or equivalent dose of other corticosteroids). Regarding alcohol consumption, the use of 3 units of alcohol or more per day was considered affirmative, in accordance with the Frax guidelines. A unit of alcohol varies little between different countries and is between $8-10 \mathrm{~g}$ of alcohol. This is equivalent to a standard glass of beer $(285 \mathrm{ml})$, a measure of spirits $(30 \mathrm{ml})$, an average glass of wine $(120 \mathrm{ml})$ or a measure of aperitif $(60 \mathrm{ml})^{16}$.

For the definition of secondary osteoporosis, Frax considers whether the patient has a disease strongly associated with osteoporosis. This includes type I diabetes, osteogenesis imperfecta in adults, untreated hyperthyroidism, hypogonadism or premature menopause ( $<45$ years), chronic malnutrition or malabsorption, and chronic liver disease ${ }^{16}$.

According to the sample of the Sapori study, postmenopausal hormone therapy and regular physical activity in the previous year had a protective role. The app was configured to only signal these affirmations as positive for identified continuous cases during the previous year ${ }^{13}$. In this study, those of white/Caucasian race/ethnicity were considered to be at greater risk, with the definition of race at the discretion of the clinical judgment of the health professional.
The data used by the osteoporosis adviser (OPAD) tool, a clinical decision support system developed in Iceland, were not used in the OSTEOGUIA assessment form as there were no studies on its use and validation for the Brazilian population. However the design pattern, with fields for the inclusion of numerical values of anthropometric data and yes or no option buttons for risk factor items, were adopted. The functionality of offering recommendations on lifestyle, treatment and the timing of bone densitometry exams immediately after submission of the form were also followed ${ }^{17}$.

With respect to the clinical recommendations for the adoption of preventive measures for osteoporosis, the application was based on the Clinical Protocol and Therapeutic Guidelines of Osteoporosis of the Ministry of Health (MS), Ordinance No. 451/201418, and the guidelines on prevention of falls among the elderly of the Brazilian Society of Geriatrics and Gerontology (SBGG) ${ }^{19}$.

\section{Automated Audit}

To evaluate the performance of the app, Google's automated open source Lighthouse tool was used ${ }^{20}$. This provides a comprehensive audit of all aspects of the quality of a web app by performing a series of tests on the page, and generates a performance report based on those tests, displaying flaws and recommendations for improving the application. Lighthouse was run as an extension of Chrome, auditing the items listed in Table 1.

Chart 1. Items of app audited with Lighthouse tool. Rio de Janeiro, 2017.

\begin{tabular}{|l|l|l|}
\hline Progressive Web App & Accessibility & Good Practices \\
\hline Register a service worker & Elements use attributes correctly & $\begin{array}{l}\text { Include JavaScript front-end libraries without } \\
\text { security vulnerabilities }\end{array}$ \\
\hline $\begin{array}{l}\text { Respond with } 200 \text { code when } \\
\text { off-line }\end{array}$ & $\begin{array}{l}\text { ARIA attributes follow best } \\
\text { practice }\end{array}$ & Avoid the app cache \\
\hline $\begin{array}{l}\text { Contain content when JavaScript is } \\
\text { not available }\end{array}$ & Elements have discernible names & Avoid WebSQL DB \\
\hline Use https & Elements describe content well & Use https \\
\hline $\begin{array}{l}\text { User can be asked to install the } \\
\text { web app }\end{array}$ & Elements are well structured & Use http/2 for own resources \\
\hline
\end{tabular}


Continuation of Chart 1

\begin{tabular}{|l|l|l|}
\hline Progressive Web App & Accessibility & Good Practices \\
\hline $\begin{array}{l}\text { Configured for a personalized } \\
\text { home screen }\end{array}$ & Specific page for valid language & $\begin{array}{l}\text { Use passive listeners to improve scrolling } \\
\text { performance }\end{array}$ \\
\hline $\begin{array}{l}\text { Address bar corresponds to brand } \\
\text { colors }\end{array}$ & Meta Tags used correctly & Avoid mutation events in own scripts \\
\hline $\begin{array}{l}\text { Has a }<\text { meta name }=\text { viewport }>\text { tag } \\
\text { with initial width or scale }\end{array}$ & Satisfactory color contrast \\
\hline $\begin{array}{l}\text { Content is sized correctly for } \\
\text { viewport }\end{array}$ & Avoid document.write () \\
\hline $\begin{array}{l}\text { Redirect http traffic for https } \\
\text { Page loads quickly enough in 3G }\end{array}$ & $\begin{array}{l}\text { Open external anchors using rel = noopener } \\
\text { on page load }\end{array}$ \\
\hline
\end{tabular}

ARIA= Accessible Rich Internet Applications.

APIs = Application Programming Interface.

Evaluation of app by health professionals

An evaluation of the prototype was carried out to discover the opinions of health professional users regarding the functionality, usability and reliability of the application. This model was presented and made available for use to the professionals of the Care Centre for the Elderly (NAI) of the Universidade do Estado do Rio de Janeiro (Uerj), from August to September 2017.

After the evaluation period, an online questionnaire was created using the Likert Scale with a score of 1 to 5 , varying from totally disagree to fully agree. Scores over 4 were considered adequate. The volunteers evaluating OSTEOGUIA were required to answer questions that are generally used in software engineering to assess the technical quality of applications. These issues were based on studies that assess features such as the functionality, reliability, usability and efficiency of mobile apps ${ }^{21}$.

The questionnaire included a question about the professional profile of the evaluators, who could be doctors, nurses, other health professionals or caregivers. For the validation of the online questionnaire, we sought to comply with NBR ISO/ IEC 25062: 2011 of the Brazilian Association of Technical Standards (ABNT) ${ }^{22}$, which recommends a minimum sampling of eight participants in the test stage.

After the evaluation phase of the prototype and corrections of the initial version, the application was sent for registration with the Institute of Technological Innovation of Uerj. Upon completion of this process, the app will be made available for free access on a secure hosting server on the Google Play Store.

\section{Research Ethics}

The development of this app followed the Guidelines and Norms Regulating Research Involving Human Beings, in accordance with the recommendations of resolution 466/2012 of the National Health Council. The project was approved by the Research Ethics Committee of the Universidade Estadual do Rio de Janeiro (Protocol No. 2706/17). 
The volunteers were previously informed about the evaluation of the application, with only those who agreed to and signed the Free and Informed Consent Form allowed to participate.

\section{RESULTS}

Three screens were created for the OSTEOGUIA app, based on the data extracted using the Osteorisk, Sapori and Frax tools for sociodemographic data (age, gender, weight, height and ethnicity), health (use of glucocorticoids, therapy hormone replacement therapy, rheumatoid arthritis, secondary osteoporosis, previous low-impact fracture, parents with a history of hip fracture) and health-related behaviors (physical activity, alcohol intake, and smoking).
The assessment form screen was based on the design used by the OPAD tool with fields for the numerical completion of the age, weight, height and T-score values and yes or no option buttons for the risk factors to be evaluated.

The guidelines on osteoporosis and prevention of falls among the elderly of the Ministry of Health and the Brazilian Society of Geriatrics and Gerontology, respectively, were used as a basis for the guidelines relating to clinical recommendations.

The home screen features a fixed menu at the top that leads to five sections: three with interactive information on osteoporosis, risk factors, and densitometry; one that leads to the assessment form and one to the contact form (Figure 1).

\begin{tabular}{|l|}
\hline \multicolumn{1}{|c|}{ OSTEOGUIA } \\
\hline Osteoporosis \\
\hline Risk Factors \\
\hline Densitometry \\
\hline Assessment Form \\
\hline Contact \\
\hline Back \\
\hline
\end{tabular}

Figure 1. Reproduction of OSTEOGUIA app screen. Rio de Janeiro, 2017.

The Osteoporosis section provides interactive buttons that lead to screens with information on definition, etiology, epidemiology, mortality, falls, and a button with a tutorial on using the app in which a link to the bibliographic references is located.

The Risk Factors section details each important risk factor in the assessment of the patient, separated into two columns: modifiable and non-modifiable factors. Each risk factor is represented by a button that provides detailed information, including important data to be considered when completing the assessment form.

As the diagnosis of osteoporosis can also be made based on the measurement of bone mineral density by the dual X-ray densitometry technique, the Densitometry section provides recommendations for the examination, according to the guidelines of the Ministry of Health.
In the Assessment Form the health professional inserts the general details of the patient and the items related to the risk factors. (Figure 2).

The patient's general data is entered in the text fields and risk factors are selected using the yes or no options, following the design pattern of the OPAD tool, and then automatically transferred to the app algorithms. If the patient has undergone a bone densitometry exam of the lumbar spine, a field asks them to provide the numeric value of the T-Score obtained in the lumbar segment of L1-L4.

After submitting the assessment form the user is directed to the final screen, Results and Clinical Recommendations. Here a report provides the BMI of the patient, with an alert for underweight patients, the risk classification of the patient for low bone density and the densitometry result as classified by the World Health Organization (WHO), categorized as osteoporosis, low bone density (osteopenia) or normal (Figure 3). 


\begin{tabular}{|c|c|}
\hline \multicolumn{2}{|c|}{ OSTEOGUIA } \\
\hline \multicolumn{2}{|l|}{ General data: } \\
\hline \multicolumn{2}{|l|}{ Age: } \\
\hline Weight: & Height: \\
\hline \multicolumn{2}{|l|}{ Gender: } \\
\hline \multicolumn{2}{|l|}{ Risk factors } \\
\hline \multicolumn{2}{|c|}{$\begin{array}{l}\text { 1- White/Caucasian race/ethnicity } \\
\text { o No } \quad \text { O Yes }\end{array}$} \\
\hline \multicolumn{2}{|l|}{ 2- Current smoker } \\
\hline 3- Three or more units of alcohol per day & alcohol per day \\
\hline o No $\quad$ o Yes & \\
\hline 5- Use of glucocorticoids & \\
\hline \multicolumn{2}{|c|}{ 6- Hormone replacement therapy } \\
\hline 7- Rheumatoid arthritis & \\
\hline \multicolumn{2}{|c|}{ 8- Secondary osteoporosis } \\
\hline \multicolumn{2}{|c|}{ 9- Previous low impact fracture } \\
\hline \multicolumn{2}{|c|}{$\begin{array}{l}\text { 10- Parents with history of hip fracture } \\
\text { o No } \quad \text { O Yes }\end{array}$} \\
\hline \multicolumn{2}{|l|}{ Densitometry } \\
\hline \multicolumn{2}{|l|}{ T-Score lumbar spine: } \\
\hline Back & \\
\hline
\end{tabular}

Figure 2. Reproduction of OSTEOGUIA app screen. Rio de Janeiro, 2017.

\begin{tabular}{|l|}
\hline \multicolumn{1}{|c|}{ OSTEOGUIA } \\
\hline \multicolumn{1}{|c|}{ Results and Clinical Recommendations } \\
\hline $\begin{array}{l}\text { 1- Body mass index BMI=17.2 } \\
\text { Being underweight is a risk factor for osteoporosis }\end{array}$ \\
\hline $\begin{array}{l}\text { 2- Classification of risk of patient for low bone density } \\
\text { = High Risk }\end{array}$ \\
\hline $\begin{array}{l}\text { 3- Result of densitometry exam, according to WHO } \\
\text { classification = Osteoporosis }\end{array}$ \\
\hline $\begin{array}{l}\text { 4- Smoking and excessive alcohol intake should be } \\
\text { discouraged }\end{array}$ \\
\hline $\begin{array}{l}\text { 5- Rheumatoid arthritis is associated with low bone } \\
\text { mass }\end{array}$ \\
\hline $\begin{array}{l}\text { 6- Use of glucocorticoid with a dose greater than } 5 \mathrm{mg} \\
\text { prednisone/day or equivalent for a period of } 3 \text { months } \\
\text { or more has a higher predictive value for fracture risk }\end{array}$ \\
\hline 7- No other modifiable risk factors \\
\hline Back \\
\hline
\end{tabular}

Figure 3. Reproduction of OSTEOGUIA app screen. Rio de Janeiro, 2017. 
In the Contact section the user is provided with a form so that they can send a message to the app developers with questions, comments and suggestions.

In the automated audit, the app presented a performance of $82 \%$ in PWA characteristics, $89 \%$ in accessibility features and $100 \%$ in complying with good web development practices. Suggested indicators for improving accessibility relate to technical features that make content accessible to a user of assistive technology, such as a screen reader for the visually impaired. Improvements to the PWA features include improvements in server access such as https access redirection, which is a secure http, and improved image optimization for a better $3 \mathrm{G}$ connection experience.

Table 1 shows the data based on the responses of the evaluations performed by NAI/UERJ professionals. All seven evaluated items were approved, with an emphasis on the ease of use and reliability of the application, and $62.5 \%$ of professionals agreed with the use of the app. Eight professionals participated in this evaluation, and all identified themselves as doctors. The online questionnaire was available in the same trial period as the prototype.

Table 1. Responses to on-line questionnaire for the assessment of the OSTEOGUIA app. Rio de Janeiro, 2017.

\begin{tabular}{lll}
\hline Items & Questions & Mean (standard-deviation) \\
\hline 1 & Would you like to use this app frequently in your day to day routine? & $4.62( \pm 0.51)$ \\
2 & Is the app easy to use? & $5.00( \pm 0)$ \\
3 & Could you use the app alone? & $5.00( \pm 0)$ \\
4 & Are the functions of the app well integrated? & $4.62( \pm 0.51)$ \\
5 & Are the functions of the app consistent? & $4.50( \pm 1.41)$ \\
6 & $\begin{array}{l}\text { Do you think other people will be able to learn } \\
\text { to use OSTEOGUIA quickly and easily? }\end{array}$ & $4.75( \pm 0.46)$ \\
7 & Do you think the use of the app is reliable? & $5.00( \pm 0)$ \\
\hline
\end{tabular}

\section{DISCUSSION}

In the daily clinical practice of elderly health care, it has been observed that many patients only receive care and treatment for osteoporosis after a fall episode in which a fragility fracture is found. Danachi et al. ${ }^{9}$ identified that simple and effective measures, such as early diagnosis, the treatment of osteoporosis and the regular practice of physical activities, were not adopted for elderly patients with a fracture of the proximal third of the femur.

Despite the many treatments available for the prevention and management of osteoporosis, with the effective reduction of vertebral and non-vertebral injuries, the majority of the Brazilian population still do not have access to early diagnosis and appropriate therapy for the disease ${ }^{8}$. The Brazilian government provides medication for osteoporosis through the SUS but, without adequate epidemiological knowledge, the implementation of public health programs is impaired ${ }^{7}$.

Research released by the Brazilian Society of Clinical Medicine showed that around $72 \%$ to $93 \%$ of physicians said they were very interested in receiving information concerning the prevention and treatment of osteoporosis. At undergraduate or graduate level, $64 \%$ and $79 \%$ of students, respectively, had little or no exposure to osteoporosis. Only $22 \%$ of physicians reported having knowledge about the guidelines for the treatment and prevention of osteoporosis ${ }^{23}$.

The OSTEOGUIA application was developed to try to fill this gap, seeking to identify the needs of health professionals and providing scientifically based information, aiming to provide a better service to the population which presents risk factors associated with osteoporosis. 
The systematic review found that there are few clinical decision support applications in osteoporosis available and that most studies evaluating these tools do not incorporate the three main components in the assessment: risk factors, diagnosis and treatment ${ }^{24}$.

Some tools, such as Osteorisk, Sapori and Frax, identify individuals at greater risk for fracture, optimizing the start of and adherence to treatment, resulting in a more effective selection of patients to undergo bone densitometry, as the doctor can request the examination for those who are most at risk by quantitatively weighing the clinical risk factors. In the $S$ core study ${ }^{13}$, the authors showed that unnecessary requests for bone densitometry could be reduced by up to $30 \%$, and the Belgian Osiris (Osteoporosis Index Risk) study showed that recommendations for this test could be reduced by $55 \%$ if the instrument was correctly applied, avoiding its use among patients at low risk ${ }^{13}$.

The OSTEOGUIA app incorporates the risk factors used by these tools with the advantages of portability and easy installation on mobile devices, adapting to any screen size by adopting a responsive design pattern. During the literature review, no online application for the use of the Osteorisk tool was identified. The Sapori tool was developed as an Excel tool and although an internet version of the Frax tool is available, the mobile app is not free.

The use of free coding tools allowed the development of a low cost app. In addition, the use of established web languages facilitates the maintenance and improvement of the app, which can be expanded by linking the assessment form to the registry in a structured database, allowing a more accurate statistical analysis with the possibility of developing new risk assessment tools that consider regional ethnic differences. The impact generated by the use of these tools in the long term and the analysis of the profile of patients in question is a possibility for extending this study.

Another feature that could be improved in the app is the provision of a statistical analysis correlating the risk factors and results of lumbar bone densitometry values with a representative population sample for the definition of sensitivity and specificity in identifying risk for osteoporosis. There is a tendency to develop such apps based on patient experience. In the next stage of this study, it is hoped that these users participate in the evaluation of OSTEOGUIA.

This progressive web app follows the new trend for the mobile web in its relative ease of development and user experience advantages, having the potential to meet the demand of health professionals working with osteoporosis.

As limitations of the present study, it should be noted that the online questionnaire applied to health professionals for the evaluation of the OSTEOGUIA app lacks a protocol for internal validation by the information technology sector of the institution. In addition, the low number of evaluators and the fact that only health professionals responded further limits the extrapolation of the results of this research.

\section{CONCLUSION}

The evaluation of the app created in terms of usability, functionality and reliability suggest the possible incorporation of this tool in the daily practice of health professionals. The OSTEOGUIA app enables the early identification of patients presenting risk factors for osteoporosis. Based on these results, the professional can provide guidance about the preventive measures to be adopted, aimed at a better quality of life, reducing complications such as fractures, hospitalizations, disabilities and deaths.

It should be emphasized that the clinical recommendations provided by the app are based on the Clinical Protocol and Therapeutic Guidelines for Osteoporosis of the Ministry of Health and the guidelines on the prevention of falls among the elderly of the Brazilian Society of Geriatrics and Gerontology. Therefore, although this app is easy to use and can be accessed by mobile devices, its guidelines are scientifically based. The implementation of the OSTEOGUIA app can bring benefits for the prevention of this disease in Brazil, allowing the reduction of the socioeconomic impact to the health services. 


\section{REFERENCES}

1. Marinho BCG, Guerra LP, Drummond JB, Silva BC, Soares MMS. The burden of osteoporosis in Brazil. Arq Bras Endocrinol Metab. 2014;58(5):434-43.

2. Tibes CMDS, Dias JD, Zem-Mascarenhas SH. Aplicativos móveis desenvolvidos para a área da saúde no Brasil: revisão integrativa da literatura. Rev Min Enferm. 2014;18(2):471-86.

3. Méndez-Sanz R, de la Torre-Díez I, López-Coronado $\mathrm{M}$. What is your risk of contracting Alzheimer's disease?: A telematics tool helps you to predict it. J Med Syst. 2016;40(1):1-9.

4. Lara MA, Tiburcio M, Aguilar Abrego A, SánchezSolís A. A four-year experience with a Web-based selfhelp intervention for depressive symptoms in Mexico. Rev Panam Salud Pública. 2014;35(5-6):399-406.

5. dos Santos TS, Alves Brito T, Yokoyama Filho FS, Guimarães LA, Souto CS, Souza SJN, et al. Desenvolvimento de aplicativo para dispositivos móveis voltado para identificação do fenótipo de fragilidade em idosos. Rev Bras Geriatr Gerontol. 2017;20(1):63-73.

6. Vêscovi SDJB, Primo CC, Sant'Anna HC, de Oliveira Bringuete ME, Rohr RV, do Prado TN, et al. Aplicativo móvel para avaliação dos pés de pessoas com diabetes mellitus. Acta Paul Enferm. 2017;30(6):607-13.

7. Baccaro LF, Conde DM, Costa-Paiva L, PintoNeto AM. The epidemiology and management of postmenopausal osteoporosis: a viewpoint from Brazil. Clin Interv Aging. 2015;10:583-91.

8. Pinheiro MM, Eis SR. Epidemiology of osteoporotic fractures in Brazil: what we have and what we need. Arq Brasil Endocrinol Metabol. 2010;54(2):164-70.

9. Daniachi D, dos Santos NA, Ono NK, Guimarães RP, Polesello GC, Honda EK. Epidemiologia das fraturas do terço proximal do fêmur em pacientes idosos. Rev Bras Ortop. 2015;50(4):371-7.

10. Chen SJ, Chen YJ, Cheng CH, Hwang HF, Chen CY, Lin MR. Comparisons of different screening tools for identifying fracture/osteoporosis risk among community-dwelling older people. Medicine. 2016;95(20):1-10.

11. Sen SS, Rives VP, Messina OD, Morales-Torres J, Riera G, Angulo-Solimano JM, et al. A risk assessment tool (OsteoRisk) for identifying Latin American women with osteoporosis. J Gen Int Med. 2005;20(3):245-50.
12. Steiner ML, Fernandes CE, Strufaldi R, Porto EC, Pompei LD, Peixoto S. Application of Osteorisk to postmenopausal patients with osteoporosis. Sao Paulo Med J. 2010;128(1):24-9.

13. Pinheiro MM, Reis Neto ET, Machado FS, Omura F, Szejnfeld J, Szejnfeld VL. Development and validation of a tool for identifying women with low bone mineral density and low-impact fractures: the São Paulo Osteoporosis Risk Index (SAPORI). Osteoporosis Int. 2012;23(4):1371-9.

14. Bastos-Silva Y, Aguiar LB, Pinto-Neto AM, Baccaro LF, Costa-Paiva L. Correlation between osteoporotic fracture risk in Brazilian postmenopausal women calculated using the FRAX with and without the inclusion of bone densitometry data. Arch Osteoporosis. 2016;11(1):1-7.

15. Daniel Nations. What is a web application? [Internet]. [Sem Local]: Lifewire; 2016 [acesso em 17 jan. 2017]. Disponível em: https://www.lifewire.com/what-is-aweb-application-3486637

16. Kanis JA, McCloskey EV, Johansson H, Oden A, Ström O, Borgström F. Development and use of FRAX ${ }^{\circledR}$ in osteoporosis. Osteoporosis Int. 2010;21(2):407-13.

17. Halldorsson BV, Bjornsson AH, Gudmundsson HT, Birgisson EO, Ludviksson BR, Gudbjornsson B. A clinical decision support system for the diagnosis, fracture risks and treatment of osteoporosis. Comp Math Meth Med. 2015;1-7.

18. Brasil. Portaria SAS/MS n ${ }^{\circ} 451$, de 9 de junho de 2014. Aprova o Protocolo Clínico e Diretrizes Terapêuticas da Osteoporose. Diário Oficial da União. 2014; Seção 1. p. 48.

19. Buksman S, Vilela ALS, Pereira SRM, Lino VS, Santos VH. Quedas em Idosos: prevenção [Internet]. Rio de Janeiro: SBGG; 2008 [acesso em 17 jan. 2017]. (Projeto Diretrizes). Disponível em: https://sbgg.org. br//wp-content/uploads/2014/10/queda-idosos.pdf

20. Tools for Web Developers. Auditar apps da Web com o Lighthouse [Internet]. [Sem Local]: Developers Google; 2017 [acesso em 17 jan. 2017]. Disponível em: https://developers.google.com/web/tools/ lighthouse/?hl=pt-br

21. Tibes CM. Aplicativo móvel para prevenção e classificação de úlceras por pressão [Dissertação]. São Carlos: Universidade Federal de São Carlos; 2015. 


\section{Associação Brasileira de Normas Técnicas. ABNT} NBR ISO/IEC 25062:2011. Engenharia de Software - Requisitos e avaliação de qualidade de produto de software (SQuaRe): Formato comum da Indústria (FCI) para relatórios de teste de usabilidade [Internet]. Rio de Janeiro: ABNT; 2011[acesso 17 jan. 2017]. Disponível em: http://www.abntcatalogo.com.br
23. Szejnfeld VL, Jennings F, Castro CH, Pinheiro MD, Lopes AC. Conhecimento dos médicos clínicos do Brasil sobre as estratégias de prevenção e tratamento da osteoporose. Rev Bras Reumatol. 2007;47(4):251-7.

24. Kastner M, Li J, Lottridge D, Marquez C, Newton D, Straus SE. Development of a prototype clinical decision support tool for osteoporosis disease management: a qualitative study of focus groups. BMC Med Inform Decis Mak. 2010;10(1):1-19.

Received: November 29, 2017

Reviewed: April 17, 2018

Accepted: July 02, 2018 